\title{
Transient Infrared Measurement of Laser Absorption Properties of Porous Materials
}

\author{
Andrzej Marynowicz \\ Department of Physics of Materials, Faculty of Civil Engineering, Opole University of Technology, ul. Katowicka 48, \\ 45-061 Opole, Poland, e-mail: a.marynowicz@po.opole.pl
}

\begin{abstract}
The infrared thermography measurements of porous building materials have become more frequent in recent years. Many accompanying techniques for the thermal field generation have been developed, including one based on laser radiation. This work presents a simple optimization technique for estimation of the laser beam absorption for selected porous building materials, namely clinker brick and cement mortar. The transient temperature measurements were performed with the use of infrared camera during laser-induced heating-up of the samples' surfaces. As the results, the absorbed fractions of the incident laser beam together with its shape parameter are reported.
\end{abstract}

Keywords: Infrared measurements, laser heating-up, energy absorption, effective beam radius, porous materials.

\section{INTRODUCTION}

Infrared thermography is a tool increasingly used within the investigations of physical properties of building materials. It has unique properties, resulting from the ability of performing contactless measurements of the whole of the investigated surface in a very short time. The intensive investigations of the internal structure of porous building materials are carried out with the use of infrared thermography, including crack formation [1], salinity of material [2], moisture migration problems [3], [4], [5], [6], [7], [8] or murals damage evaluation [9]. The separate group of investigations concern thermal properties estimation of the materials. In the work of Bison et al. [10] an interesting approach, utilizing the laser impulse method, has been described. In the proposed method, the authors recorded the resulting thermal response of the sample surface by an infrared camera. In further analysis, based on the Fourier transform of the recorded image, the studied thermal diffusivity has been evaluated.

The most numerous group of currently investigated materials are the ones with uniform internal structure, with small spatial variation of material properties, for example metals, pure ceramics and plastics [11], [12], [13], [14]. Another separate group is represented by building porous materials with mostly non-homogenous structure, such as ceramic brick, aerated concrete (AAC) [15], cement and lime mortar, and concrete [2], [3], [10]. These materials generate additional problems during infrared testing. Among them, one can find unidentified impurities (for example in ceramic bricks) and inclusions of different origin, such as aggregates in mortars and certain clinker bricks. In the proposed approach, the influence of the aforementioned inclusions has been reduced by the approximation of the measured temperature field with the pseudo-Voight function.

The main objective of the presented work was to evaluate the applicability of the infrared camera in order to estimate a laser beam absorption by the surfaces of selected porous building materials. The shape of the laser beam spot was also studied, due to better description of the boundary condition in the analyzed case.

With a thermovision camera operating principles in mind, it was necessary to produce a required temperature field in the modeled nonstationary thermal process. In this area more or less complicated methods have been developed, for example with the use of surface heaters [16], a thermal impulse with constant intensity [17], hot air [18], or heating lamps [19].

To simplify the experiment, the infrared camera, together with the low-cost, low-power, red CW laser module, have been used. This assembly was used for estimation of the energy absorption fraction and the shape parameter of an incident laser beam, during the short time of continuous sample heating. The knowledge of these two quantities can be used in further research for the estimation of the thermal properties of porous building materials with a similar structure. 


\section{MATERIALS \& METHODS}

\subsection{Description of the problem}

To achieve the goals featured in the introduction, the classical two-dimensional heat transfer model with the cylindrical symmetry was assumed (Fig.1.a), described by the differential equation

$$
\frac{\rho c_{p}}{\lambda} \frac{\partial T}{\partial t}=\frac{\partial^{2} T}{\partial r^{2}}+\frac{1}{r} \frac{\partial T}{\partial r}+\frac{\partial^{2} T}{\partial z^{2}},
$$

with the initial condition $T=T_{0}$ in the whole area occupied by the body (for $t=0$ ) and the boundary conditions (see Fig.1.b)

$$
\begin{aligned}
& -\left.\lambda \frac{\partial T}{\partial z}\right|_{z=0}=q_{\text {las }}(r)-h_{z}\left(T-T_{e}\right) \quad \text { for } \quad z=0, \\
& -\left.\lambda \frac{\partial T}{\partial r}\right|_{r=N}=h_{r}\left(T-T_{e}\right) \quad \text { for } \quad r=N, \\
& -\left.\lambda \frac{\partial T}{\partial z}\right|_{z=M}=h_{z}\left(T-T_{e}\right) \quad \text { for } \quad z=M,
\end{aligned}
$$

where $q_{\text {las }}(r)$ describes the absorbed part of an incident laser beam [11], [12], [13], expressed as

$$
q_{\text {las }}(r)=\frac{2 Q_{a b b}}{\pi R_{e f f}^{2}} e^{-\frac{2 r^{2}}{R_{e f f}^{2}}} .
$$

The quantities $Q_{a b s}$ and $R_{e f f}$ are treated in a similar way, like the ones in the formula describing a laser beam in the technological processes based on laser treatment of materials, but this time $Q_{a b s}$ denotes the power of the absorbed laser beam, and $R_{\text {eff }}$ is the effective Gaussian (for which $I=I_{0} e^{-2}$ [13]). The assumption of boundary condition in the form of (2a) and (3) is justified by the very small depth of the transitional zone, in which the laser beam transfers into the heat energy, with estimated order of several dozens of micrometers [20], [21].

For the simplification of the computational model, it is assumed that heat flux is "shifted" by the width of that transitional zone. The above approach, implying that $Q_{a b s}$ and $R_{\text {eff }}$ depend on material properties, is motivated by the high superficial property variations (especially roughness) and a different internal structure of porous building materials. This may change the range of the laser beam interaction with the material. The above assumption will be discussed in section 3 . The parameters appearing in equation (3), i.e. $R_{\text {eff }}$ and $Q_{a b s}$, are necessary for correct modeling of the initial-boundary problem, described by (1) and (2). As mentioned above, the problem lies in the fact that these quantities depend on surface properties of material. Let us assume that a thin transitional zone behaves like a surface heat source. Then, we should firstly determine the properties of that source in the experiment.
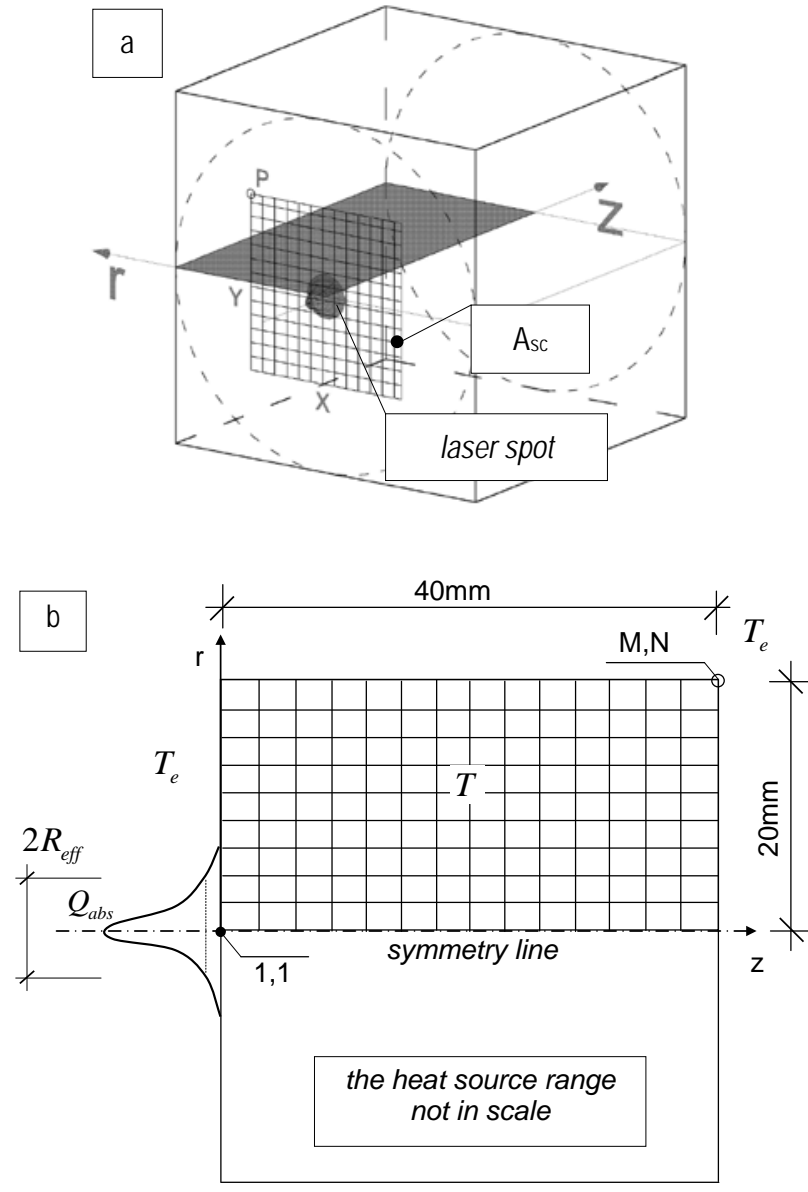

Fig.1. Sample geometry a), and its computational model b).

The unknown parameters, i.e. $\mathbf{u}=\left[Q_{a b s}, R_{e f f}\right]$, were estimated using the method of minimization of objective function $F(\mathbf{u})$ given in the form

$$
\mathbf{u}_{0}=\arg \min _{\mathbf{u}} F(\mathbf{u})=\sum_{i=1}^{n}\left[\left.Y_{i}\left(r_{i}, t\right)\right|_{z=0}-\left.T_{i}\left(\mathbf{u}, r_{i}, t\right)\right|_{z=0}\right]^{2},
$$

where $Y_{i}\left(r_{i}, t\right)$ denotes the vector of temperature measured at the surface $(\mathrm{z}=0)$ at the $i$-th point and the time $t$. The vector of temperature $T_{i}\left(\mathbf{u}, r_{i}, t\right)$ was calculated at the same points, with the specified set of parameters $\mathbf{u}$. The values of $T_{i}$ elements were calculated from (1), with boundary conditions (2), by the means of the two-dimensional Finite Difference Method (later called FDM), according to the Peaceman - Rachford implicit ADI scheme [12], [22], [23]. The ADI technique is based on the unconditionally stable, implicit solution of the problem in separate directions. The analyzed domain has been divided into equally sized elements in both directions. The elements have the dimensions of $0.305 \times 0.305 \mathrm{~mm}$, what corresponds to the size of the area seen by the one pixel of IR camera. For further analysis, only the part of sample surface, denoted $A_{s c}$ (Fig.1.a.), was taken into account, due to a relatively small laser spot range. 
The simplification of the sample geometry model, described above, was justified by the fact that the time consuming 3D analysis of the same domain led to very similar results. For the comparison of the two models, the 3D finite difference scheme was developed. The differences in the resulting surface temperature between 2D and 3D models were negligible. Therefore, it has been assumed that the proposed simplification is suitable for further analysis.

\subsection{Materials used and test assembly}

The measurements of temperature values $Y_{i}(r, t)$ at the sample surface were performed for two different materials: clinker brick with sand admixture (CK) and cement mortar (ZC), made according to the code EN 196-1 from water, cement CEM I 32.5 and sand with $0 \div 2 \mathrm{~mm}$ aggregates, in weight proportions $0.5: 1$ : 3 . In Table 1 , the mean values of major physical properties of analyzed materials, determined in $25^{\circ} \mathrm{C}$, are summarized. The measurements of thermal properties, i.e. thermal diffusivity $a$ and thermal conductivity $\lambda$, were carried out with the use of Netzsch LFA427 analyzer (laser-flash based apparatus).

Table 1. Physical properties of tested materials (CK - clinker brick, ZC - cement mortar).

\begin{tabular}{|c|c|c|c|c|}
\hline & $a=\frac{\lambda}{\rho c_{p}}$ & $\rho\left[\frac{\mathrm{kg}}{\mathrm{m}^{3}}\right]$ & $c_{p}\left[\frac{\mathrm{J}}{\mathrm{kg} \cdot \mathrm{K}}\right]$ & $\lambda\left[\frac{\mathrm{W}}{\mathrm{m} \cdot \mathrm{K}}\right]$ \\
\hline $\mathrm{CK}$ & $0.71 \cdot 10^{-6}$ & 2175 & 790 & 1.220 \\
\hline $\mathrm{ZC}$ & $1.13 \cdot 10^{-6}$ & 1943 & 945 & 2.075 \\
\hline
\end{tabular}

In order to perform temperature measurements, during the sample surface heating-up process by the laser beam, the experimental assembly was built, as shown in Fig.2. The sample was placed on the wooden stand 1, equipped with graphite-coated shield 2 to reduce beam reflection effects. As the heat source, we used the low-power laser diode module 3 (Mitsubishi ML101J27, $660 \mathrm{~nm}$, max. $150 \mathrm{~mW}$ $\mathrm{CW}$ ), together with anamorphic prism pair 4 (Thorlabs), for the beam shape correction. As the temperature recording device 5, the VigoCAM v50 infrared camera (VIGO System, NETD $=0.08 \mathrm{~K}$, spectral range $8 \div 14 \mu \mathrm{m}$ ) was used. The sensitivity of the camera is suitable to perform the measurements in the narrow range of temperature changes. The assembly was completed with a stable laser power supply unit 7 and a PC computer 6 .

The measurements were taken in room conditions. Before the measurements the samples were stored in room temperature, with the air humidity about $\mathrm{RH}=65 \%$. The heat transfer coefficients were initially assumed to have an equal value, i.e. $h_{r}=h_{z}=7.7 \mathrm{~W} \cdot \mathrm{m}^{-2} \cdot K^{-1}$, what is usually provided for the heat exchange calculations near vertical interior planes, without forced convection. This condition is provided because of small temperature rise caused by the laser radiation. In section 3.2 the model sensitivity for these coefficients, together with further simplifications, will be discussed.

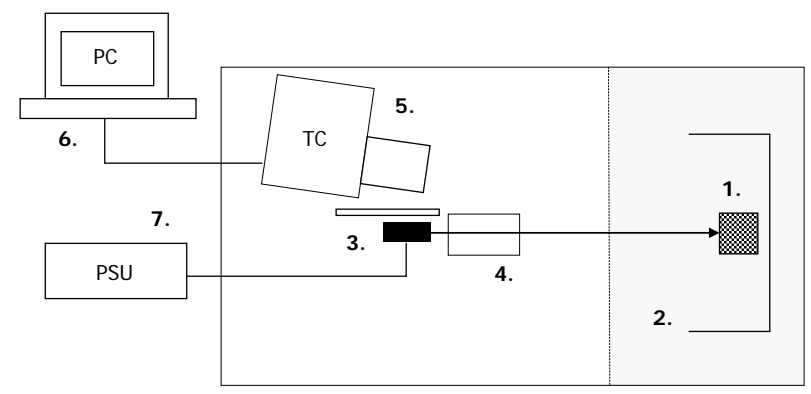

Fig.2. Test assembly scheme: 1- sample on a stand, 2- shield, 3laser module, 4- correction prisms, 5- infrared camera, 6- PC computer, 7- power supply unit.

The accuracy of the infrared temperature measurements of physically heterogeneous materials is affected with numerous errors, arising from different emissivities of individual fragments of the analyzed area. In Fig.3., the surface structure of analyzed materials is shown in the visible range, with numerous aggregate intrusions clearly visible for both CK (a) and ZC (b) samples. Furthermore, Fig.4. and Fig.5. contain the images of the same samples in infrared range, where we can see the effects of intrusions on temperature readings. These two images were taken during sample cooling, after long-time conditioning in the constant temperature near $T=70^{\circ} \mathrm{C}$.
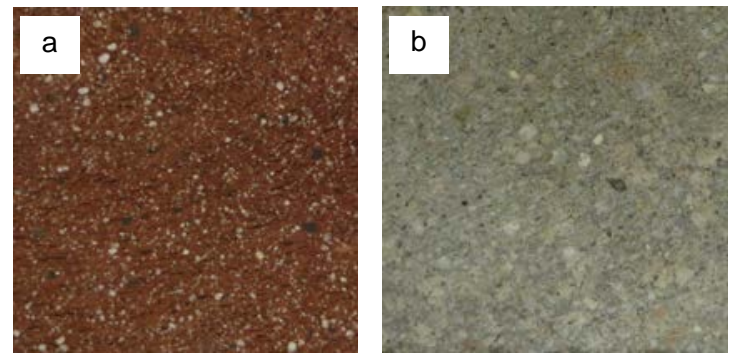

Fig.3. Surface images of analyzed samples - visible region: a) - CK, b) - ZC.

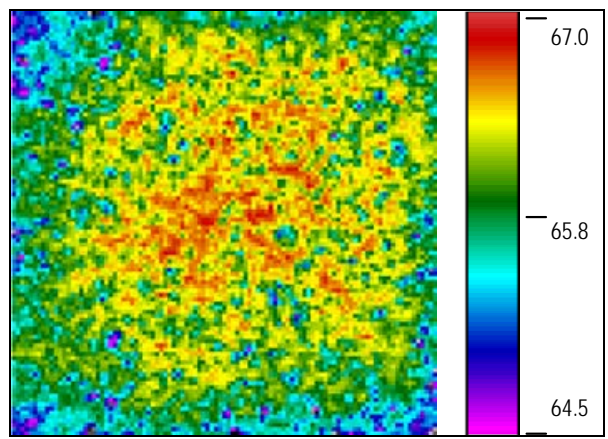

Fig.4. Infrared images of surface temperature $\left({ }^{\circ} \mathrm{C}\right)$ of the $\mathrm{CK}$ sample. 


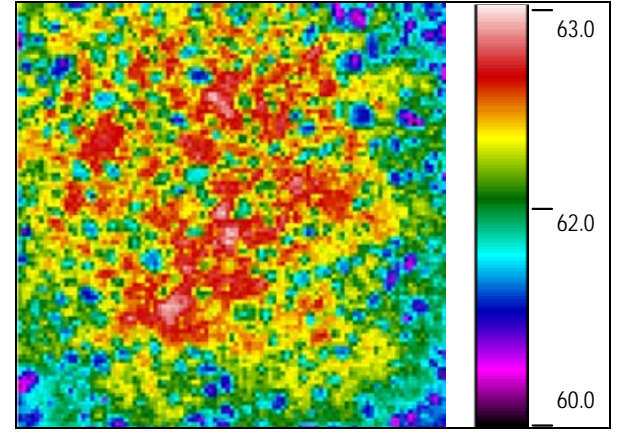

Fig.5. Infrared images of surface temperature $\left({ }^{\circ} \mathrm{C}\right)$ of the ZC sample.

\section{THE MEASUREMENT}

\subsection{Data preparation}

As we could see in section 2.1, one of the components of minimized function $F(\mathbf{u})$ is the vector of temperature $Y_{i}\left(r_{i}, t\right)$, measured in specified points and time. In order to observe temperature changes during laser heating-up of the surface, the experimentally estimated measurement time of $20 \mathrm{~s}$ was chosen. The reduction of the measurement time to $20 \mathrm{~s}$ was sufficient due to fast temperature stabilization in the center of the spot. It also allowed for elimination of the sensing matrix drift. For both tested materials, the sequence of 20 frames, from 1 to 20 s with 1s step, was recorded. Line $\mathrm{L}$, close to the spot horizontal axis was chosen as the most appropriate for further analysis, as one can see in Fig.6., Fig.7., for the clinker brick and Fig.8., Fig.9. for the cement mortar.

In addition to the reasons discussed in section 2.2, the measurements of surface temperature based on infrared imaging are also affected by noise generated by IR sensing matrix [24]. To minimize the influence of these effects on calculation precision of the functional $F(\mathbf{u})$, the global approximating pseudo-Voigt function was chosen, resulting from the convolution of Gaussian and Lorentzian functions [25], in the form

$$
\begin{aligned}
f(\mathbf{v}, r) & =y_{0}+A \cdot\left[\mu \frac{2 w}{4 \pi\left(r-r_{c}\right)^{2}+w^{2}}+\right. \\
& \left.+(1-\mu) \frac{\sqrt{4 \ln 2}}{w \sqrt{\pi}} e^{\frac{-4 \ln 2}{w^{2}}\left(r-r_{c}\right)^{2}}\right]
\end{aligned},
$$

where $\mathbf{v}=\left[y_{0}, A, w, r_{c}, \mu\right]$ denotes the vector of the function $f(\mathbf{v}, r)$ parameters, i.e. offset along y axis, amplitude, peak half-width (FWHM), location of the maximum and Lorentzian contribution, respectively. The searched parameters were calculated with the use of Nelder-Mead simplex method (the 'fminsearch' procedure of MATLAB software). The function (5) was found to be the most appropriate to describe the temperature distributions and it applied in the analyzed case. The adjusted $R^{2}$ calculations gave results from 0.996 to 0.998 , which allowed for the acceptance of fitting quality of the proposed function (5).

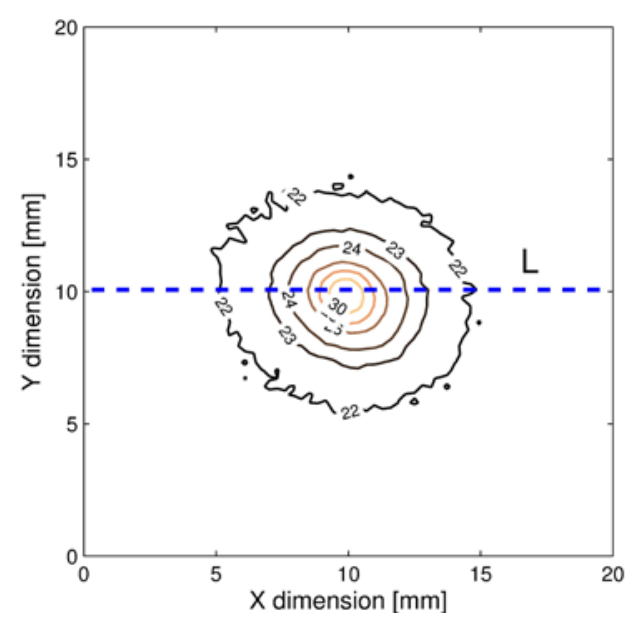

Fig.6. Temperature isolines at the surface $A_{s c}$ of CK sample after $5 \mathrm{~s}$ of heating-up.

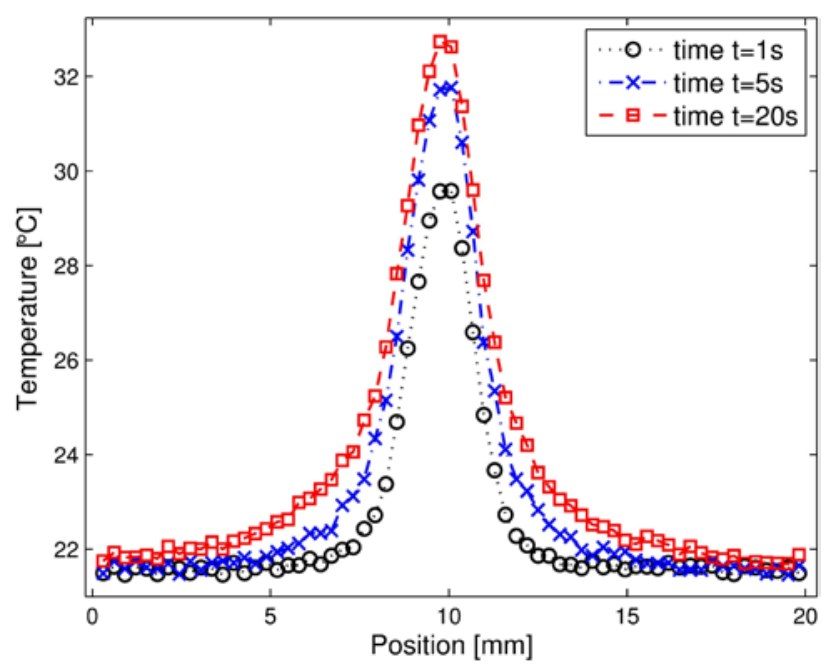

Fig.7. Temperature at line L for CK sample in selected time of process.

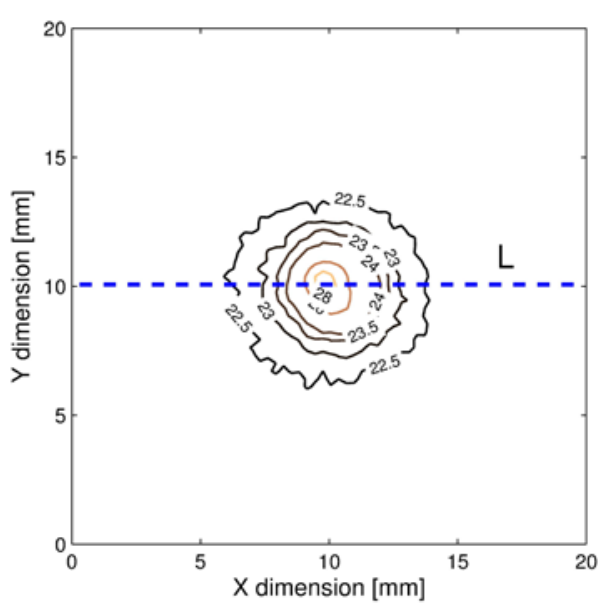

Fig.8. Temperature isolines at the surface $A_{s c}$ of ZC sample after $5 \mathrm{~s}$ of heating-up. 


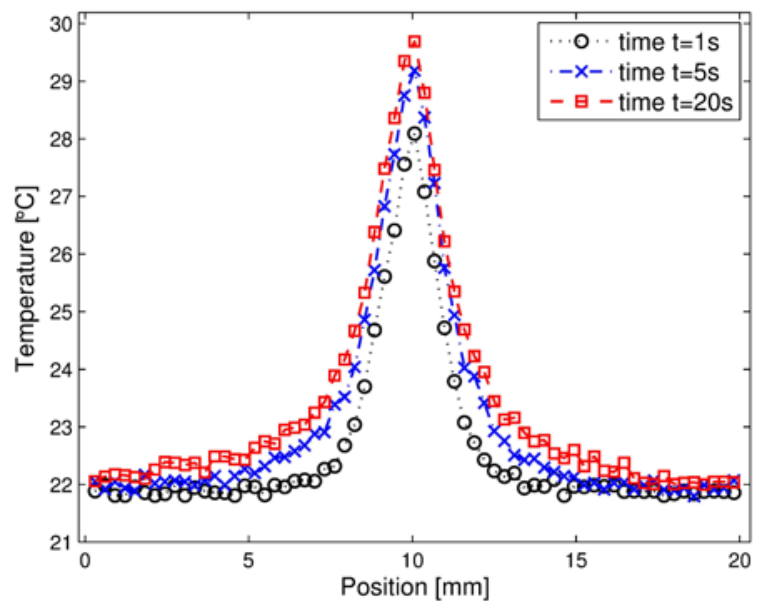

Fig.9. Temperature at line L for ZC sample in selected time of process.

In Fig.10.a) and Fig.10.b), we can observe the selected results of fitting the function (5) to the temperature measurements, for $t=1 \mathrm{~s}$ and $t=15 \mathrm{~s}$, for CK and ZC sample, respectively.
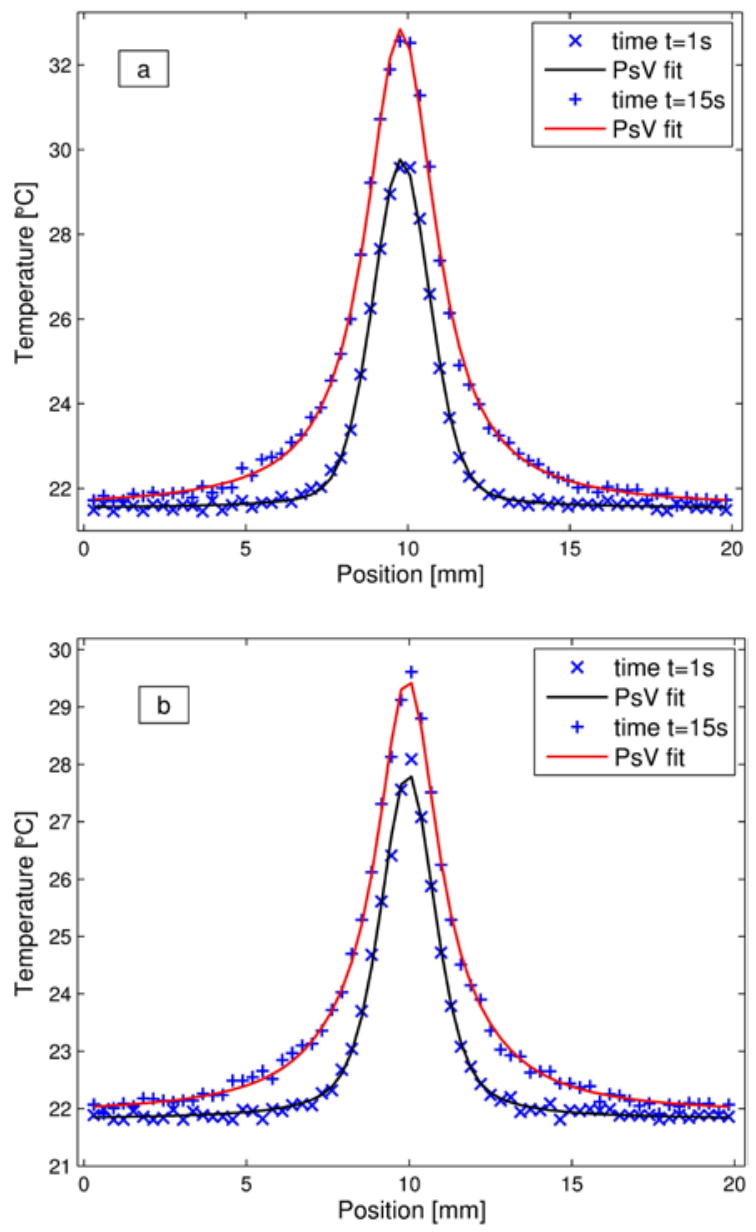

Fig.10. Selected results of pseudo-Voigt function fitting (PsV) for $\mathrm{CK}$ a) and $\mathrm{ZC} \mathrm{b}$ ) sample.

\subsection{Results of the calculations}

After replacing the temperature measurements $Y_{i}\left(r_{i}, t\right)$ with the fitting function (5), the functional $F(\mathbf{u})$ have finally the form

$$
F(\mathbf{u})=\sum_{i=1}^{n}\left[\left.f_{i}\left(\mathbf{v}, \mathrm{r}_{i}, t\right)\right|_{z=0}-\left.T_{i}(\mathbf{u}, r, t)\right|_{z=0}\right]^{2}
$$

The minimization of functional (6) was performed using the direct search method within the $\mathbf{u}$ parameter domain. After the analysis of the expected domain range, the limiting values for the effective radius were chosen as $R_{\text {eff }}=\left\{1.2 \cdot 10^{-3} \div 1.7 \cdot 10^{-3}\right\}[\mathrm{m}]$, and for the absorbed beam power as $Q_{a b s}=\left\{50 \cdot 10^{-3} \div 70 \cdot 10^{-3}\right\}[W]$. Finally, the domain ranges of $R_{\text {eff }}$ and $Q_{a b s}$ were divided into 30 and 20 steps, respectively.

Table 2. Averaged results of $\bar{Q}_{a b s}$ and $\bar{R}_{\text {eff }}$ estimation.

\begin{tabular}{|c|c|c|c|}
\hline Sample & $T_{e}=T_{0}\left[{ }^{\circ} \mathrm{C}\right]$ & $\bar{Q}_{a b s}[\mathrm{~W}]$ & $\bar{R}_{\text {eff }}[\mathrm{m}]$ \\
\hline CK & 21.7 & $59.9 \cdot 10^{-3}$ & $1.61 \cdot 10^{-3}$ \\
\hline ZC & 21.9 & $64.4 \cdot 10^{-3}$ & $1.50 \cdot 10^{-3}$ \\
\hline
\end{tabular}

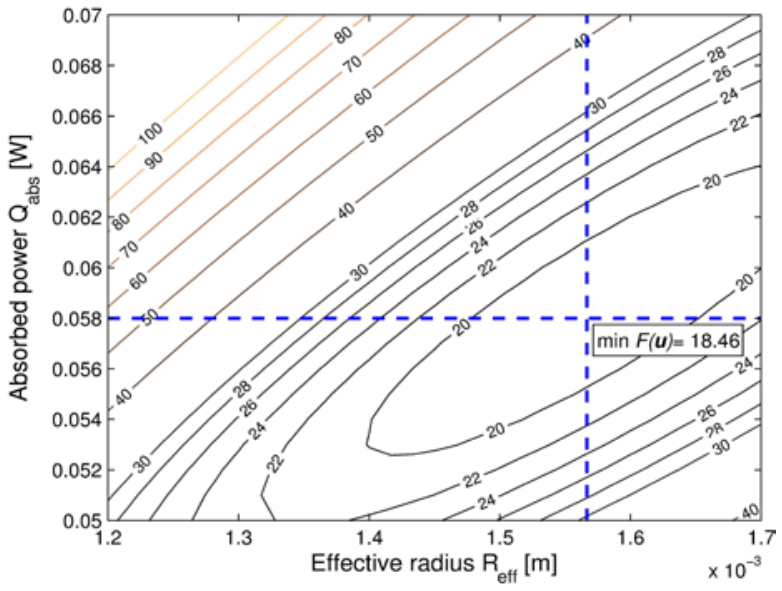

Fig.11. Error function plot of $F(\mathbf{u})$ for $t=5 s-C K$ sample.

As the result of the FDM calculations, the temperature vector $T_{i}$ for every time step was obtained. Using relation (6), the value of functional $F(\mathbf{u})$ was calculated for each of the 20 time points. Fig.11. and Fig.12. show the selected maps of $F(\mathbf{u})$ for $t=5 \mathrm{~s}$ for both tested materials, together with a position of the single minimum and corresponding values of $Q_{a b s}$ and $R_{\text {eff }}$, which proves the uniqueness of the solution. Finally, in Fig.13. and Fig.14. the resulting values are plotted together, for every time step. In Table 2., 
averaged values of examined parameters are listed. It is worth pointing out that the difference between $\bar{Q}_{a b s}$ and $\bar{R}_{\text {eff }}$ for $\mathrm{CK}$ and $\mathrm{ZC}$ sample is about $8 \%$. This difference gives us important information about the absorption abilities of the tested materials.

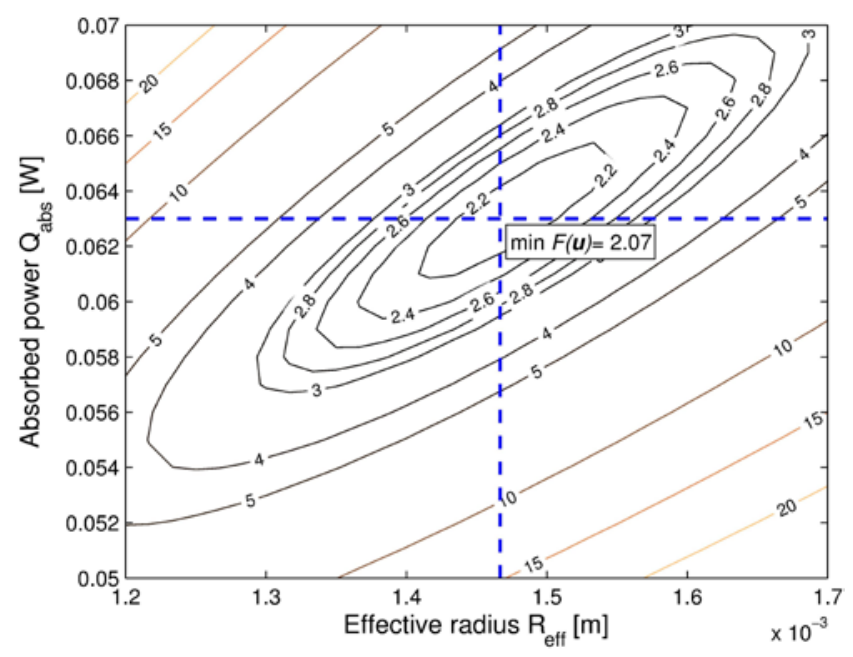

Fig.12. Error function plot of $F(\mathbf{u})$ for $\mathrm{t}=5 s-\mathrm{ZC}$ sample.

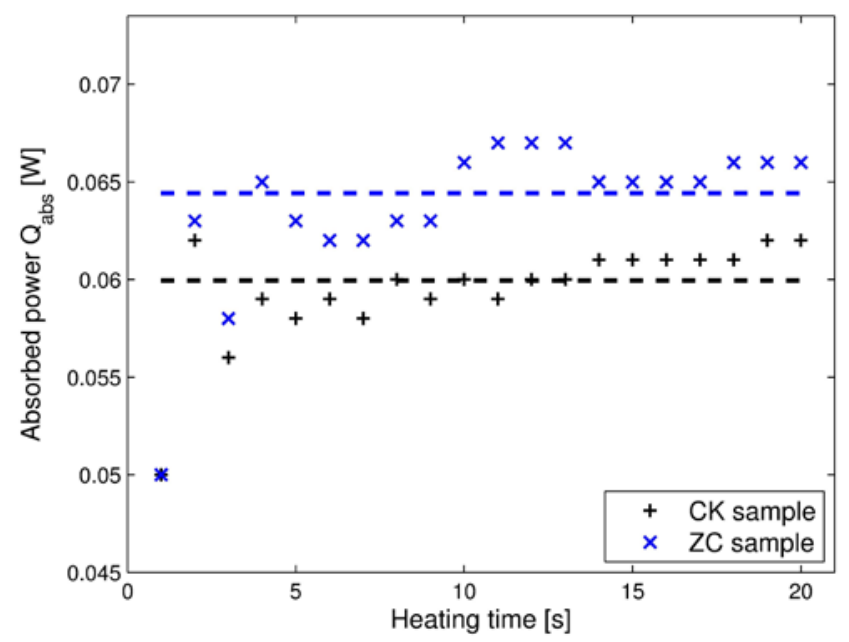

Fig.13. Estimation of the absorbed power $Q_{a b s}$. Dashed lines denote mean values (see table 2).

For the estimation of the model sensitivity for changes of heat transfer coefficient variations, the additional calculations have been performed. After the assumption that convectional flux is negligible in comparison with the laser induced surface heat source, what was possible because $h_{z}\left(T-T_{e}\right)<<\mathrm{q}_{\text {las }}[26]$, we found, that the differences between the resulting heat conductivities do not exceed $1 \%$. Therefore, the rejection of the convectional part of the boundary conditions (2a)-(2c) (setting them to the zero value) is justified for the future developments of the proposed method.

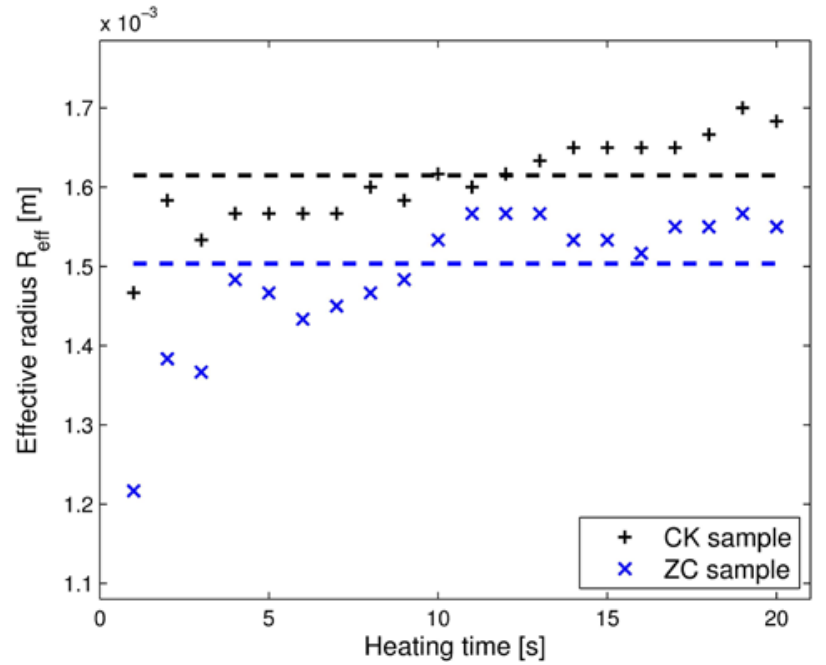

Fig.14. Estimation of the effective radius $R_{\text {eff }}$. Dashed lines denote mean values (see table 2).

\section{CONCLUSIONS}

In a comparison with other experimental methods, mentioned in the introduction, the presented methodology allows performing relatively uncomplicated and effective investigation of the surface absorption properties of porous building materials. In the first stage of the proposed approach the classical formulation of heat transfer in cylindrical coordinates was solved numerically by means of the Finite Difference Method. Thereafter, the optimization method based on the direct domain search was used for estimation of the key parameters appearing in the boundary condition of the problem. This condition is tightly bonded with the searched absorption property and laser beam shape parameter of investigated materials.

On the basis of the results achieved in the work, the following primary conclusions can be formulated:

- The proposed method gives possibility of laser absorption estimation of porous building material with a very good precision.

- The laser-induced heat source shape parameter, which can be assumed as constant one in case of homogenous materials, should be determined individually for specified groups of porous building materials.

The further developments of the proposed method give also a chance to perform the measurements of other materials' properties, for example thermal diffusivity. To achieve this goal, the database of laser absorption for the common building materials should be prepared. The influence of superficial properties of the tested samples, concerning their finishing, roughness or moisture content in a material is the other important aspect, which should be considered in the next research.

\section{ACKNOWLEDGMENT}

The IR camera equipment was partially supported by European Regional Development Fund within a framework of Operational Trans-Border-Cooperation Program 20072013 Czech Republic-Poland (CZ.3.22/2.3.00/08.00635). 


\section{REFERENCES}

[1] Schlichting, J., Maierhofer, Ch., Kreutzbruck, M. (2012). Crack sizing by laser excited thermography. NDT and E International, 45, 133-140.

[2] Gayo-Moncó, E., de Frutos, J. (1998). Hydric processes associated with saline solutions, studied by means of selective infrared thermography. Cement and Concrete Research, 28, 1165-1177.

[3] Furmański, P., Wiśniewski, T.S. (2009). Determination of moisture content in a porous building material using infra-red based method. ISESCO Journal of Science and Technology, 5, 71-75.

[4] Gayo, E., de Frutos, J., Palomo, A., Massa, S. (1996). A mathematical model simulating the evaporation processes in building materials: Experimental checking through infrared thermography. Building and Environment, 31, 469-475.

[5] Lerma, C., Mas, Á., Gil, E., Vercher, J., Peñalver, M.J. (2014). Pathology of building materials in historic buildings. Relationship between laboratory testing and infrared thermography. Materiales de Construcción, 64 (313).

[6] Camino, M.S., León, F.J., Llorente, A., Olivar, J.M. (2014). Evaluation of the behavior of brick tile masonry and mortar due to capillary rise of moisture. Materiales de Construcción, 64 (314).

[7] Edis, E., Flores-Colen, I., de Brito, J. (2015). Quasiquantitative infrared thermographic detection of moisture variation in facades with adhered ceramic cladding using principal component analysis. Building and Environment, 94, 97-108.

[8] Barreira, E., Almeida, R.M.S.F., Delgado, J.M.P.Q. (2016). Infrared thermography for assessing moisture related phenomena in building components. Construction and Building Materials, 110, 251-269.

[9] Kordatos, E.Z., Exarchos, D.A., Stavrakos, C., Moropoulou, A., Matikas, T.E. (2012). Infrared thermographic inspection of murals and characterization of degradation in historic monuments. Construction and Building Materials, 48, 1261-1265.

[10] Bison, P., Bortolin, A., Cadelano, G., Ferrarini, G., Grinzato, E. (2012). Comparison of some thermographic techniques applied to thermal properties characterization of porous materials. In 11th International Conference on Quantitative InfraRed Thermography (QIRT 2012), Naples, Italy.

[11] Bison, P., Cernuschi, F., Grinzato, E., Marinetti, S., Robba, D. (2007). Ageing evaluation of thermal barrier coatings by thermal diffusivity. Infrared Physics and Technology, 49, 286-291.

[12] Ivanović, I.B., Sedmak, A.S., Miloš, M.V., Živković, A.B., Lazić, M.M. (2011). Numerical study of transient three-dimensional heat conduction problem with a moving heat source. Thermal Science, 15, $257-$ 266.
[13] Modest, M. (1996). Three-dimensional, transient model for laser machining of ablating/decomposing materials. International Journal of Heat and Mass Transfer, 39, 221-234.

[14] Zhou, J., Zhang Y., Chen, J.K., Feng, Z.C. (2010). Inverse estimation of surface heating condition in a three-dimensional object using conjugate gradient method. International Journal of Heat and Mass Transfer, 53, 2643-2654.

[15] Barreira, E., de Freitas, V.P. (2007). Evaluation of building materials using infrared thermography. Construction and Building Materials, 21, 218-224.

[16] Zmywaczyk, J., Madura, H., Koniorczyk, P., Dąbrowski, M. (2007). Estimation of thermophysical properties by an inverse method with experimentally determined heating region of a thin-layer heater. Infrared Physics and Technology, 49, 277-280.

[17] Perkowski, Z. (2011). A thermal diffusivity determination method using thermography: Theoretical background and verification. International Journal of Heat and Mass Transfer, 54, 2126-2135.

[18] Cerdeira, F., Vázquez, M.E., Collazo, J., Granada, E. (2011). Applicability of infrared thermography to the study of the behaviour of stone panels as building envelopes. Energy \& Buildings, 43, 1845-1851.

[19] Grinzato, E., Vavilov, V., Kauppinen, T. (1998). Quantitative infrared thermography in buildings. Energy and Buildings, 29, 1-9.

[20] Wojtatowicz, T. (ed.) (2000). Investigations of Phase Transitions in Surface Layer of Porous Body with Use of Laser Irradiation. Łódź, Poland: Politechnika Łódzka. (in Polish)

[21] Li, J.F., Li, L., Stott, F.H. (2004). Comparison of volumetric and surface heating sources in the modeling of laser melting of ceramic materials. International Journal of Heat and Mass Transfer 47, 1159-1174.

[22] Wang, T.-Y., Chen, C.-P. (2003). Thermal-ADI - a linear-time chip-level dynamic thermal-simulation algorithm based on alternating-direction-implicit (ADI) method. IEEE Transactions on Very Large Scale Integration (VLSI) Systems, 11 (4), 691-700.

[23] Ozisik, M.N. (1994). Finite Difference Methods in Heat Transfer. CRC Press, p. 151.

[24] Minkina, W., Dudzik, S. (2009). Infrared Thermography: Errors and Uncertainties. Wiley, p. 32.

[25] Jansson, P. (ed.) (1984). Deconvolution: With Applications in Spectroscopy. Academic Press, p. 10.

[26] Dowden, J. (ed.) (2009). The Theory of Laser Materials Processing. Springer, p.79.

Received November 10, 2015. Accepted May 27, 2016. 